\title{
Smoking and Periodontitis Can Play a Synergistic Role in the Development of Psoriasis: A Nationwide Cohort Study
}

\author{
Ju Hee Han ${ }^{a}$ Jin Woo Park ${ }^{a}$ Kyung Do Han ${ }^{b}$ Jun Beom Park ${ }^{c}$ Miri Kim ${ }^{d}$ \\ Ji Hyun Lee ${ }^{a}$ \\ aDepartment of Dermatology, Seoul St. Mary's Hospital, College of Medicine, The Catholic University of Korea, \\ Seoul, Republic of Korea; bepartment of Statistics and Actuarial Science, Soongsil University, Seoul, Republic of \\ Korea; 'Department of Periodontics, Seoul St. Mary's Hospital, College of Medicine, The Catholic University of Korea, \\ Seoul, Republic of Korea; 'Department of Dermatology, Yeouido St. Mary's Hospital, College of Medicine, The \\ Catholic University of Korea, Seoul, Republic of Korea
}

\author{
Keywords \\ Epidemiology · Periodontitis · Prevalence · Psoriasis . \\ Smoking
}

\begin{abstract}
Background: Periodontitis is a chronic inflammatory disorder involving the periodontium. The precise nature of the association between periodontitis and psoriasis has not been determined. Objective: This nationwide populationbased study investigated the relationship between periodontitis and the risk of psoriasis. Methods: A health screening database, which is a sub-dataset of the Korean National Health Insurance System database, was used in this study. Subjects with ( $n=1,063,004)$ and without $(n=8,655,587)$ periodontitis who underwent health examinations from January to December 2009 were followed for 9 years. Results: In multivariable analysis, compared to the non-periodontitis group, periodontitis patients had a significantly higher risk of developing psoriasis (hazard ratio 1.116, 95\% confidence interval 1.101-1.13). Non-smokers with periodontitis had an $11 \%$ increase in risk of psoriasis and smokers with periodon-
\end{abstract}

karger@karger.com www.karger.com/drm

Karger $\stackrel{\text { ' }}{5}$

BOPEN ACCESS
(C) 2021 The Author(s)

Published by S. Karger AG, Basel

This is an Open Access article licensed under the Creative Commons Attribution-NonCommercial-4.0 International License (CC BY-NC) (http://www.karger.com/Services/OpenAccessLicense), applicable to the online version of the article only. Usage and distribution for commercial purposes requires written permission. titis had a $26.5 \%$ increase in risk of psoriasis compared to non-smokers without periodontitis. Conclusion: Our study highlights periodontitis as a potential independent risk factor for psoriasis, increasing awareness of the synergistic role of smoking and periodontitis in the pathogenesis of psoriasis.

(c) 2021 The Author(s).

Published by S. Karger AG, Basel

\section{Introduction}

Psoriasis is a chronic inflammatory disorder with an estimated prevalence of $1-3 \%$ in the general population $[1,2]$. Psoriasis may involve skin, nails, and joints. Moreover, it is related with various systemic comorbidities such as cardiovascular disease (CVD), obesity, and metabolic syndrome $[1,3,4]$.

Periodontitis is a chronic inflammatory disorder involving the periodontium [5]. Some recent studies have

Reprint requests: M. Kim.
Correspondence to:

Miri Kim, mirikim@ catholic.ac.kr

Ji Hyun Lee, ejee@ catholic.ac.kr 


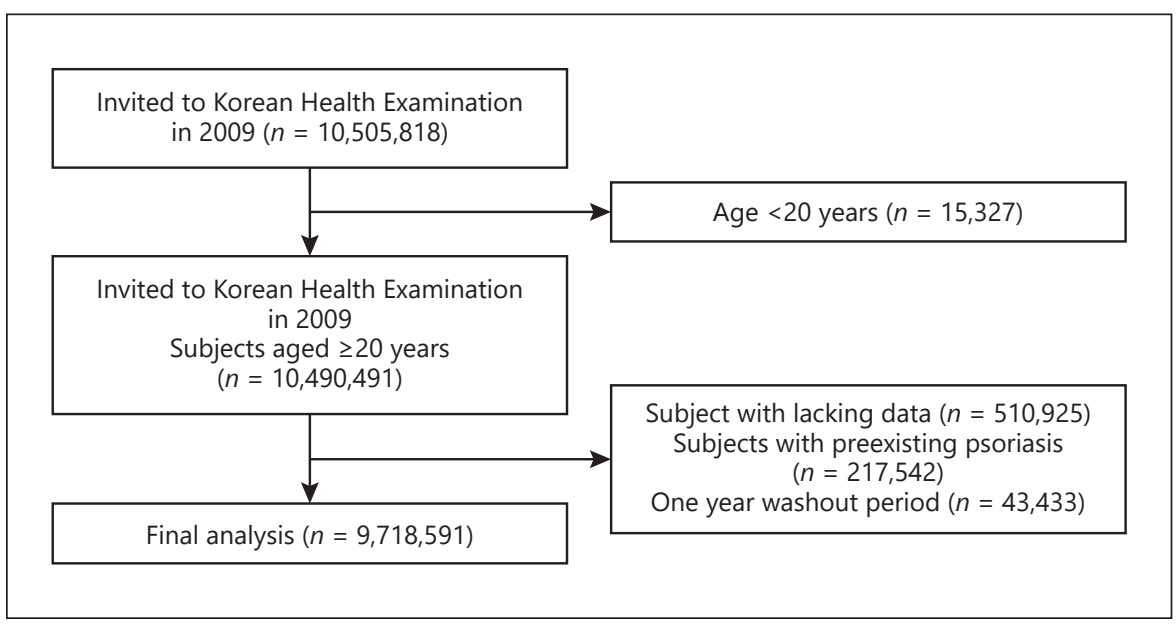

Fig. 1. Flowchart of included patients.

assessed the associations between periodontitis and immune-mediated systemic inflammatory disorders [6-8]. Bacterial colonization in the oral cavity may induce an exaggerated immune response in a susceptible host, leading to an inflammatory process associated with autoimmune disorders $[6,9,10]$. Indeed, patients with periodontitis tend to have higher incidence and prevalence rates of obesity, diabetes mellitus, CVD, and rheumatoid arthritis [9,11-13]. Although several previous studies have suggested a link between periodontitis and psoriasis, the precise nature of the association and its underlying mechanism have not been determined [7, 14, 15]. Moreover, no large-scale, nationwide cohort studies have examined whether periodontitis increases the risk of psoriasis. Therefore, we investigated the relationship between periodontitis and risk of psoriasis using the $\mathrm{Na}$ tional Health Insurance System (NHIS) claims database in Korea.

\section{Materials and Methods}

\section{Data Source}

The representative health screening database of the Korean NHIS was used in this study. The NHIS is a single, essential health insurance program that covers approximately $97 \%$ of the Korean population. The remaining 3\% of the population with low income is covered by a medical aid program, and their data have been integrated into the NHIS database [16]. Therefore, since 2006, the NHIS database actually includes the whole Korean population. Health checkups are managed by the government and are implemented by almost all of the Korean population. NHIS members are encouraged to undergo a health checkup every 2 years. Patient demographic information and medical claims are included in the NHIS database. The database is based on the International Clas- sification of Disease, Tenth Revision, Clinical Modification (ICD10-CM) codes.

\section{Study Population}

From the NHIS database, patients aged $\geq 20$ years who underwent health screening from January 2009 to December 2009 were included in the study. We applied a 12-month washout period to exclude patients with prediagnosed periodontitis and psoriasis. Patients were excluded if they were $<20$ years of age, were missing data, or were diagnosed with psoriasis prior to the health screening. Ultimately, 1,063,004 subjects with periodontitis and 8,655,587 subjects without periodontitis and a total 9,718,591 subjects were followed for 9 years from January 2009 to December 2017 (Fig. 1). We identified patients with newly diagnosed psoriasis during the follow-up period, based on the claims data. Patients with periodontitis and psoriasis were identified using the ICD-10-CM codes (chronic periodontitis [K05.3], psoriasis [L40]). Periodontitis is usually treated through scaling, subgingival curettage, or periodontal surgery based on the depth of the periodontal pocket and bone destruction, and is covered by the NHIS in Korea. Therefore, the periodontitis group was divided into three stages, according to the severity of periodontitis, based on therapeutic procedures including scaling, subgingival curettage, and periodontal surgery $[17,18]$.

\section{Data and Baseline Comorbidities}

Information on height, weight, waist circumference, systolic blood pressure, diastolic blood pressure, fasting plasma glucose, total cholesterol, low-density lipoprotein levels, highdensity lipoprotein levels, body mass index (BMI, $\mathrm{kg} / \mathrm{m}^{2}$ ), smoking status (non-smoker, ex-smoker, or current smoker), alcohol consumption (non-drinker, mild drinker [ $<30 \mathrm{~g} /$ day], or heavy drinker [ $\geq 30 \mathrm{~g} /$ day]), physical activity, and socioeconomic status was obtained from the health screening program. Comorbid diseases were defined according to both diagnostic codes and associated prescribed medication during the study period [16]. 
Table 1. Characteristics of the study population

\begin{tabular}{llll}
\hline & No periodontitis & Periodontitis & $p$ value \\
\hline Number & $8,655,587$ & $1,063,004$ & $<0.0001$ \\
Sex & & & \\
$\quad$ Male & $4,727,593(54.62 \%)$ & $585,936(55.12 \%)$ & $<0.0001$ \\
$\quad$ Female & $3,927,994(45.38 \%)$ & $477,068(44.88 \%)$ & $<0.0001$ \\
Current smoker & $2,286,772(26.42 \%)$ & $251,458(23.66 \%)$ & $<0.0001$ \\
Heavy drinker & $595,649(6.88 \%)$ & $69,501(6.54 \%)$ & $<0.0001$ \\
Physical activity (regular) & $4,440,450(51.3 \%)$ & $556,876(52.39 \%)$ & $<0.0001$ \\
Low income & $1,803,830(20.84 \%)$ & $214,307(20.16 \%)$ & $<0.0001$ \\
Comorbidities & & & $<0.0001$ \\
$\quad$ Diabetes mellitus & $712,667(8.23 \%)$ & $127,249(11.97 \%)$ & $<0.0001$ \\
$\quad$ Hypertension & $2,160,780(24.96 \%)$ & $328,066(30.86 \%)$ & $<0.0001$ \\
$\quad$ Hyperlipidemia & $1,531,076(17.69 \%)$ & $236,480(22.25 \%)$ & $<0.0001$ \\
Age, years & $46.55 \pm 14.14$ & $51.36 \pm 12.9$ & $<0.0001$ \\
BMI, kg/m & $23.68 \pm 3.22$ & $23.91 \pm 3.09$ & $<0.0001$ \\
WC, cm & $80.09 \pm 9.09$ & $81.14 \pm 8.84$ & $<0.0001$ \\
SBP, mm Hg & $122.32 \pm 14.94$ & $123.09 \pm 15$ & $<0.0001$ \\
DBP, mm Hg & $76.26 \pm 9.97$ & $76.56 \pm 9.96$ & $<0.0001$ \\
FPG, mg/dL & $96.79 \pm 22.52$ & $99.57 \pm 25.29$ & $<0.0001$ \\
TC, mg/dL & $194.77 \pm 36.52$ & $196.8 \pm 36.98$ & $117.71(117.59-117.84)$ \\
TGa mg/dL & $113.05(113.01-113.1)$ & $28.47(28.43-28.51)$ & \\
Y-GTPa, IU/L & $27.38(27.37-27.39)$ & & \\
\hline
\end{tabular}

Data are expressed as mean (standard deviation) for continuous variables and $n$ (\%) for binary variables. Statistical analyses were performed by comparing clinical characteristics between patients with or without periodontitis using Student $t$ test for continuous variables and $x^{2}$ test for categorical variables. BMI, body mass index; DBP, diastolic blood pressure; FPG, fasting plasma glucose; $\gamma$-GTP, $\gamma$-glutamyl transpeptidase; SBP, systolic blood pressure; TC, total cholesterol; TG, triglyceride; WC, waist circumference. ${ }^{\text {a }}$ Data were log-transformed before analysis.

Statistical Analyses

The baseline characteristics of the study population are expressed as numbers and percentages or means \pm standard deviations. Differences in clinical characteristics according to the presence of psoriasis were analyzed using Student $t$ test or $\chi^{2}$ test. To evaluate the risk of incident psoriasis, Cox proportional hazards regression analyses were performed to calculate the hazard ratio (HR) and 95\% confidence interval (CI). After adjustment for demographic factors, the multivariable $\mathrm{HR}$ and 95\% CI were calculated. Model 1 was adjusted for age and sex. Model 2 was adjusted for age, sex, alcohol consumption, physical activity, BMI, diabetes mellitus, hypertension, dyslipidemia, and income level. Subgroup analyses were performed according to periodontitis and smoking status. The study population was divided into the following four groups: no periodontitis with/ without smoking, and periodontitis with/without smoking. Cumulative incidence curves were plotted using the Kaplan-Meier method and statistical significance was examined using the log-rank test. Statistical analyses were performed using SAS software version 9.4 (SAS Institute, Cary, NC, USA), and two-sided $p<0.05$ was considered to indicate statistical significance.

\section{Results}

Baseline Characteristics of the Study Population

From January 2009 to December 2009, we identified $1,063,004$ patients with periodontitis and $8,655,587$ patients without. The baseline and clinical characteristics of the study population are summarized in Table 1.

\section{Risk of Psoriasis in Periodontitis Patients}

Table 2 shows the incidence rates of psoriasis and risk of psoriasis according to the presence of periodontitis. The incidence rates of psoriasis per 1,000 person-years were 3.477 in patients with periodontitis and 2.904 in the non-periodontitis group. In multivariable analysis, compared to the non-periodontitis group, patients with periodontitis (HR 1.116, 95\% CI 1.101-1.13) had a significantly higher risk of developing psoriasis. However, subgroup analysis according to periodontitis severity showed no difference in psoriasis risk. 
Table 2. Risks and incidence rates of psoriasis in patients with periodontitis

\begin{tabular}{|c|c|c|c|c|c|c|c|}
\hline No & $8,655,587$ & 180,565 & 2.90362 & 1 (ref.) & & 1 (ref.) & \\
\hline Yes & $1,063,004$ & 26,494 & 3.47745 & $1.117(1.103,1.132)$ & & $1.116(1.101,1.13)$ & \\
\hline Severity of periodontitis & & & & & $<0.0001$ & & $<0.0001$ \\
\hline Moderate & 243,770 & 6,019 & 3.433 & $1.114(1.086,1.143)$ & & $1.113(1.085,1.142)$ & \\
\hline Severe & 34,494 & 822 & 3.31632 & $1.076(1.005,1.152)$ & & $1.077(1.005,1.153)$ & \\
\hline
\end{tabular}

Data are expressed as hazard ratios and 95\% confidence intervals. ${ }^{\mathrm{a}}$ Model 1 was adjusted for age and sex. ${ }^{\mathrm{b}}$ Model 2 was adjusted for age, sex, smoking status, alcohol drinking status, exercise status, household income, BMI, and comorbidities of type 2 diabetes mellitus, hypertension, and hyperlipidemia.

Table 3. Risks and incidence rates of psoriasis in each subgroup, stratified according to periodontitis and smoking status

\begin{tabular}{|c|c|c|c|c|}
\hline Group & $n$ & Psoriasis & $\begin{array}{l}\text { Incidence per } \\
1,000 \text { person-years }\end{array}$ & Model $2^{\mathrm{a}}$ \\
\hline No periodontitis, non-smoker & $6,368,815$ & 129,480 & 2.82462 & 1 (ref.) \\
\hline No periodontitis, smoker & $2,286,772$ & 51,085 & 3.12515 & $1.118(1.105,1.131)$ \\
\hline Periodontitis, non-smoker & 811,546 & 19,621 & 3.36481 & $1.11(1.094,1.127)$ \\
\hline Periodontitis, smoker & 251,458 & 6,873 & 3.8449 & $1.265(1.234,1.296)$ \\
\hline
\end{tabular}

Data are expressed as hazard ratios and $95 \%$ confidence intervals. ${ }^{\text {a }}$ Model 2 was adjusted for age, sex, smoking status, alcohol drinking status, exercise status, household income, BMI, and comorbidities of type 2 diabetes mellitus, hypertension, and hyperlipidemia.

\section{Risk of Psoriasis in Subgroup Depending on}

Periodontitis and Smoking

Table 3 shows the results of multivariable Cox proportional hazards regression analysis for the association between each subgroup and incident psoriasis, classified into four subgroups according to presence of periodontitis and smoking status. Non-smokers with periodontitis exhibited an $11 \%$ increase in the risk of psoriasis (HR $1.11,95 \%$ CI 1.094-1.127) compared to non-smokers without periodontitis, whereas smokers with periodontitis exhibited a $26.5 \%$ increase in the risk of psoriasis (HR $1.265,95 \%$ CI 1.234-1.296) compared to non-smokers without periodontitis.

\section{Cumulative Incidence Rates of Psoriasis}

Kaplan-Meier analysis revealed that the cumulative incidence rate of psoriasis was significantly higher in patients with periodontitis than in the non-periodontitis group throughout the entire study period (log-rank test, $p<0.0001$ ) (Fig. 2).

\section{Discussion}

This nationwide population-based cohort study evaluated the association between periodontitis and the risk of psoriasis. We found a significantly increased risk of incident psoriasis among patients with periodontitis compared to patients without. Moreover, the risk of psoriasis was significantly higher in the periodontitis/smoker group than in the non-periodontitis/smoker and periodontitis/non-smoker groups. The cumulative incidence rate of psoriasis was significantly higher in patients with periodontitis than in patients without periodontitis throughout the entire study period. 
Consistent with our findings, two previous meta-analysis studies demonstrated that periodontitis was associated with a 1.55 -fold (pooled relative risk 1.55, 95\% CI $1.35-1.77$ ) and 2.87-fold (pooled relative risk $2.87,95 \% \mathrm{CI}$ $1.75-4.69$ ) increased risk of psoriasis compared to the absence of periodontitis $[6,19]$. Among the studies included in previous meta-analysis studies were reports conducted in Asia (Taiwan) and Western countries (United States, Norway, Greece, and Italy), all of which support that periodontitis increases the risk of psoriasis [20-24]. Similarly, a cohort study in Taiwan found a higher risk of psoriasis in patients with periodontitis (HR 1.52, 95\% CI 1.38-1.70) compared to non-periodontitis subjects [20]. In addition, there is a previous report suggesting the association between periodontal bone loss and the risk of psoriasis [21]. Epidemiological studies have recently reported a link between periodontitis and psoriasis, and several studies have reported that severe psoriasis may increase the severity and risk of periodontitis [7, 22, 25-27]. A longitudinal cohort study of the Danish population found an increased risk of periodontitis in patients with psoriasis, with the highest risk found in patients with psoriatic arthritis [7]. Moreover, the severity of periodontitis has been shown to be correlated with the severity of psoriasis [25]. However, few studies have examined the severity of psoriasis according to the severity of periodontitis. Most of these studies were conducted with small numbers of patients through meta-analyses, cohort studies, and case-control studies, and the severity was often unclassified or classified into two stages, and some were based on self-reported severity $[6,20,21,25]$. In this study, subgroup analysis according to periodontitis severity showed no difference in psoriasis risk. This study differs from previous studies in that it is a large population-based study and classified the severity of periodontitis into three stages based on therapeutic procedures, such as scaling, subgingival curettage, and periodontal surgery. Although the classification of the severity of periodontitis according to treatment method has not yet been clearly verified, it is meaningful in that it reflects the severity in the actual treatment environment, and further validation of severity classification might be needed.

Although the exact mechanism by which periodontitis acts as a risk factor for psoriasis has not been identified, there is increasing evidence that periodontitis and psoriasis share several common immunopathogenic processes $[14,25]$. First, the inflammatory environments in periodontitis and psoriasis share many similarities. Studies have suggested that Porphyromonas gingivalis is the main pathogen of periodontitis and that it activates innate and

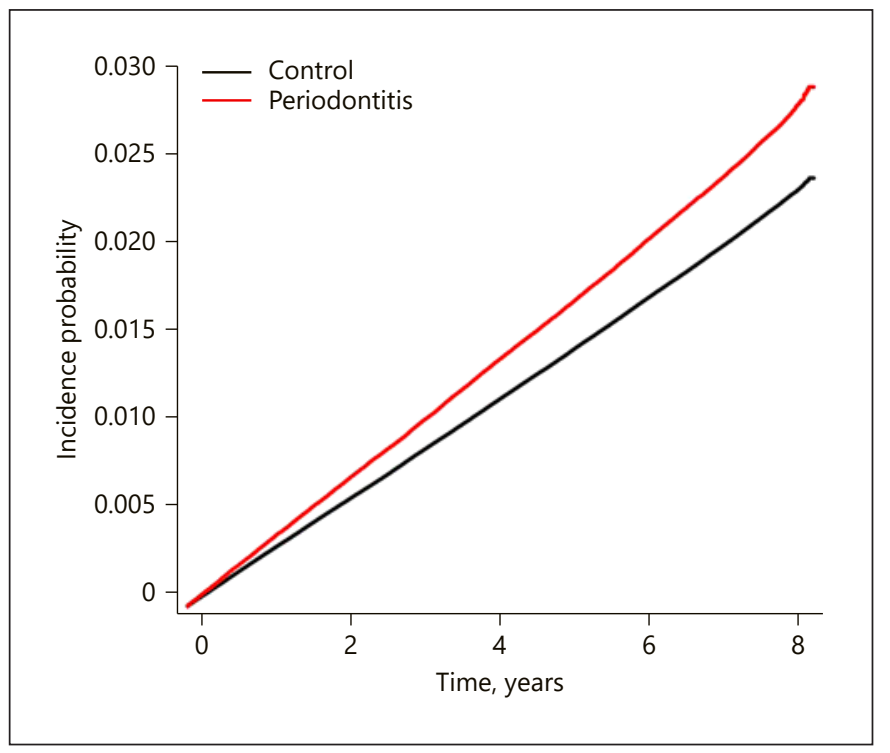

Fig. 2. Kaplan-Meier estimates of the cumulative incidence rates of psoriasis $(p<0.0001)$.

adaptive immune responses. It is also suggested that in periodontitis, immune activation by dysbiotic pathogens may trigger a systemic inflammatory reaction similar to psoriasis $[7,28]$. The microenvironment in periodontitis induces proinflammatory cytokines, such as tumor necrosis factor alpha, interleukin (IL)-1 $\beta$, and IL-17, and reduces IL-4 and Il-10, which is also observed in psoriasis $[14,22,26,29]$.

Second, periodontitis and psoriasis share common risk factors and comorbidities, and it is thought that this may serve as a link between the two diseases [30, 31]. Several reports suggest that patients with periodontitis exhibit an increased risks of metabolic syndrome and CVD, which is a common comorbidity in psoriasis $[8,13,30,32$, 33]. Chronic periodontitis has been suggested to be a distant source of inflammatory byproducts associated with insulin resistance and obesity $[13,34,35]$. The association between metabolic syndrome and periodontitis consists of a constant low-grade inflammatory status, which can lead to the development of insulin resistance and imbalanced interactions between cytokines and the periodontium [34]. In addition, there is emerging evidence that bacterial infection and modulation of inflammation are key elements in the development of atherogenesis, which causes CVD [32, 33]. Antibodies against periodontal pathogens react with host antigens and cause systemic inflammation and endothelial dysfunction [33]. In patients with periodontitis mastication, tooth brushing, and 
dental intervention could promote bacteremia and systemic low-grade inflammation. As a result, proinflammatory cytokines such as tumor necrosis factor alpha, IL-1 $\beta$, and IL- 6 can enter the circulatory system and promote atherogenesis and obesity $[33,36]$.

In this study, smoking periodontitis patients had a $26.5 \%$ higher risk of psoriasis compared with the nonperiodontitis non-smoking group. Furthermore, among patients with periodontitis, the risk of psoriasis increased significantly in smokers compared to non-smokers. Studies have shown that smoking is an independent risk factor for psoriasis. It has been suggested to also be a risk factor for periodontitis through effects on microbiota composition, immune response, and periodontal healing [14, 3739]. In addition, smoking is considered a major risk factor for refractory periodontitis, and heavy smoking is considered a risk factor for progression of periodontitis after treatment $[40,41]$. The systemic effects of smoking on chronic inflammatory diseases such as rheumatoid arthritis, multiple sclerosis, CVD, inflammatory bowel disease, periodontitis, and psoriasis have been assessed in several studies [42-45]. Smoking has been reported to cause decreased leukocyte chemotaxis and immunoglobulin production, impaired phagocytosis and inflammatory response, and increased secretion of tissue-destructive substances [42, 46, 47]. Moreover, in chronic inflammatory diseases, gene-environment interactions increase disease susceptibility $[42,48]$. In short, smoking, a common factor exacerbating periodontitis and psoriasis, might have triggered a synergistic effect on the occurrence of psoriasis. Our results show that periodontitis is a potential independent risk factor for psoriasis. Moreover, periodontitis and smoking may synergistically promote the development of psoriasis. This study is meaningful in that it analyzed the effects of periodontitis in psoriasis in a large population. Furthermore, it demonstrated the synergistic effect of periodontitis and smoking on the development of psoriasis.

There are several limitations to this study. (1) The diagnoses of periodontitis and psoriasis were conducted on the basis of the NHIS claim data, without direct review of medical records. Also, NHIS database records lack detailed information regarding the severity and type of psoriasis and family history of the study population. Diagnosis in the NHIS claim database is performed by a dentist for periodontitis and a dermatologist for psoriasis, respectively. However, the possibility of misclassification bias due to the limitation of the claim database must be considered. (2) Some patients included in this study might have had psoriasis that was not pre- viously diagnosed. In addition, since only patients who visited the hospital and were diagnosed were included in the study, patients who did not visit the hospital were not included, and there is a possibility of selection bias. Moreover, it is difficult to exclude the possibility that reverse association may have occurred due to previously undiagnosed psoriasis patients. (3) Health checkups are managed by the government and are implemented by NHIS subscribers, which is almost all of the Korean population. They are recommended to undergo health checkups every 2 years, and the data are entered into the health checkup database. Although almost all of the Korean population are included in the health checkup database, it is difficult to exclude the possibility that some healthy patients might not access the health checkup system. Therefore, a kind of semi-"healthy user bias" should be considered.

Despite these limitations, the major strengths of this study are its large sample size and nationally representative patient population. In addition, the database used is maintained by the government and public organizations that provide national health information, which makes the data stable. Finally, we controlled for confounding factors such as BMI, smoking status, alcohol consumption status, and physical activity level.

In conclusion, our study highlights periodontitis as a potential independent risk factor for psoriasis, increasing the awareness of the role of periodontitis in the pathogenesis and comorbidities of psoriasis. The results suggest that periodontitis and smoking may synergistically increase the risk of psoriasis. Patients with periodontitis should be monitored for psoriasis, and increased focus on lifestyle correction may be warranted.

\section{Key Message}

Periodontitis is a potential independent risk factor for psoriasis, and periodontitis and smoking may synergistically increase the risk of psoriasis.

\section{Statement of Ethics}

The authors have no ethical conflicts to declare. This study was approved by the Ethics Committee of Seoul St. Mary's Hospital, the Catholic University of Korea (IRB No. KC20ZISI0510), and was conducted in accordance with the principles of the Declaration of Helsinki. 


\section{Conflict of Interest Statement}

The authors have no conflicts of interest to declare.

\section{Funding Sources}

This study was supported by a National Research Foundation of Korea (NRF) grant funded by the Korean government (MSIP) (No. 2018R1D1A1B07044100).

\section{Author Contributions}

Design and conduct of the study: J.H. Han and K.D. Han. Collection, management, analysis, and interpretation of data: K.D. Han and J.B. Park. Preparation, review, or approval of the manuscript: J.H. Han, J.W. Park, M. Kim, and J.H. Lee. Decision to submit the manuscript for publication: M. Kim and J.H. Lee.

\section{References}

1 Snekvik I, Smith CH, Nilsen TIL, Langan SM, Modalsli EH, Romundstad PR, et al. Obesity, waist circumference, weight change, and risk of incident psoriasis: prospective data from the HUNT study. J Invest Dermatol. 2017; 137(12):2484-90.

2 Oh EH, Ro YS, Kim JE. Epidemiology and cardiovascular comorbidities in patients with psoriasis: a Korean nationwide populationbased cohort study. J Dermatol. 2017;44(6): 621-9.

3 Tollefson MM, van Houten HK, Asante D, Yao X, Maradit Kremers H. Association of psoriasis with comorbidity development in children with psoriasis. JAMA Dermatol. 2018;154(3):286-92.

4 Kim ES, Han K, Kim MK, Park YM, Baek KH, Moon SD, et al. Impact of metabolic status on the incidence of psoriasis: a Korean nationwide cohort study. Sci Rep. 2017;7(1):1989.

5 Cheng WC, Hughes FJ, Taams LS. The presence, function and regulation of IL-17 and Th17 cells in periodontitis. J Clin Periodontol. 2014;41(6):541-9.

6 Ungprasert P, Wijarnpreecha K, Wetter DA. Periodontitis and risk of psoriasis: a systematic review and meta-analysis. J Eur Acad Dermatol Venereol. 2017;31(5):857-62.

7 Egeberg A, Mallbris L, Gislason G, Hansen PR, Mrowietz U. Risk of periodontitis in patients with psoriasis and psoriatic arthritis. J Eur Acad Dermatol Venereol. 2017;31(2): 288-93.

8 Lee CY, Kuan YH, Tsai YF, Tai CJ, Tsai TH, Huang KH. Correlation between diabetes mellitus and periodontitis in Taiwan: a nationwide cohort study. Diabetes Res Clin Pract. 2019;150:245-52.

9 Mercado FB, Marshall RI, Klestov AC, Bartold PM. Relationship between rheumatoid arthritis and periodontitis. J Periodontol. 2001;72(6):779-87.

10 Bunte K, Beikler T. Th17 cells and the IL-23/ IL-17 axis in the pathogenesis of periodontitis and immune-mediated inflammatory diseases. Int J Mol Sci. 2019;20(14):3394.

11 Painsi C, Hirtenfelder A, Lange-Asschenfeldt $\mathrm{B}$, Quehenberger F, Wolf $\mathrm{P}$. The prevalence of periodontitis is increased in psoriasis and linked to its inverse subtype. Skin Pharmacol Physiol. 2017;30(6):324-8.
12 Ryden L, Buhlin K, Ekstrand E, de Faire U, Gustafsson A, Holmer J, et al. Periodontitis increases the risk of a first myocardial infarction: a report from the PAROKRANK study. Circulation. 2016;133(6):576-83.

13 Suvan J, D'Aiuto F, Moles DR, Petrie A, Donos N. Association between overweight/obesity and periodontitis in adults. A systematic review. Obes Rev. 2011;12(5):e381-404.

14 Mendes VS, Cota LOM, Costa AA, Oliveira AMSD, Costa FO. Periodontitis as another comorbidity associated with psoriasis: a casecontrol study. J Periodontol. 2019;90(4):35866.

15 Sezer U, Şenyurt SZ, Gündoğar H, Erciyas K, Üstün K, Kimyon G, et al. Effect of chronic periodontitis on oxidative status in patients with psoriasis and psoriatic arthritis. J Periodontol. 2016;87(5):557-65.

16 Han JH, Lee JH, Han KD, Kim HN, Bang CH, Park YM, et al. Increased risk of psoriasis in subjects with abdominal obesity: a nationwide population-based study. J Dermatol. 2019;46(8):695-701.

17 Kim SJ, Kim K, Choi S, Chang J, Kim SM, Park $\mathrm{SM}$, et al. Chronic periodontitis and community-acquired pneumonia: a populationbased cohort study. BMC Pulm Med. 2019; 19(1):268

18 Huang YF, Chang CT, Liu SP, Muo CH, Tsai $\mathrm{CH}$, Hong $\mathrm{HH}$, et al. The impact of oral hygiene maintenance on the association between periodontitis and osteoporosis: a nationwide population-based cross sectional study. Medicine (Baltimore). 2016;95(6): e2348.

19 Zhang X, Gu H, Xie S, Su Y. Periodontitis in patients with psoriasis: a systematic review and meta-analysis. Oral Dis. 2020 doi: 10.1111/odi.13617. Online ahead of print.

20 Keller JJ, Lin HC. The effects of chronic periodontitis and its treatment on the subsequent risk of psoriasis. Br J Dermatol. 2012;167(6): 1338-44.

21 Nakib S, Han J, Li T, Joshipura K, Qureshi AA. Periodontal disease and risk of psoriasis among nurses in the United States. Acta Odontol Scand. 2013;71(6):1423-9.
22 Lazaridou E, Tsikrikoni A, Fotiadou C, Kyrmanidou E, Vakirlis E, Giannopoulou C, et al. Association of chronic plaque psoriasis and severe periodontitis: a hospital based casecontrol study. J Eur Acad Dermatol Venereol. 2013;27(8):967-72.

23 Skudutyte-Rysstad R, Slevolden EM, Hansen BF, Sandvik L, Preus HR. Association between moderate to severe psoriasis and periodontitis in a Scandinavian population. BMC Oral Health. 2014;14:139.

24 Ganzetti G, Campanati A, Santarelli A, Pozzi V, Molinelli E, Minnetti I, et al. Involvement of the oral cavity in psoriasis: results of a clinical study. Br J Dermatol. 2015;172(1):282-5.

25 Sharma A, Raman A, Pradeep AR. Association of chronic periodontitis and psoriasis: periodontal status with severity of psoriasis. Oral Dis. 2015;21(3):314-9.

26 Moutsopoulos NM, Kling HM, Angelov N, Jin W, Palmer RJ, Nares S, et al. Porphyromonas gingivalis promotes Th17 inducing pathways in chronic periodontitis. J Autoimmun. 2012;39(4):294-303.

27 Su NY, Huang JY, Hu CJ, Yu HC, Chang YC. Increased risk of periodontitis in patients with psoriatic disease: a nationwide population-based retrospective cohort study. PeerJ. 2017;5:e4064.

28 Christophers E. Periodontitis and risk of psoriasis: another comorbidity. J Eur Acad Dermatol Venereol. 2017;31(5):757-8.

29 Zenobia C, Hajishengallis G. Basic biology and role of interleukin-17 in immunity and inflammation. Periodontol 2000. 2015;69(1): 142-59.

30 Qiao P, Shi Q, Zhang R, E L, Wang P, Wang $\mathrm{J}$, et al. Psoriasis patients suffer from worse periodontal status - a meta-analysis. Front Med (Lausanne). 2019;6:212.

31 Gheorghita D, Antal MA, Nagy K, Kertesz A, Braunitzer G. Smoking and psoriasis as synergistic risk factors in periodontal disease. Fogorv Sz. 2016;109(4):119-24

32 Liljestrand JM, Paju S, Pietiäinen M, Buhlin $K$, Persson GR, Nieminen MS, et al. Immunologic burden links periodontitis to acute coronary syndrome. Atherosclerosis. 2018;268: $177-84$. 
33 Khumaedi AI, Purnamasari D, Wijaya IP, Soeroso Y. The relationship of diabetes, periodontitis and cardiovascular disease. Diabetes Metab Syndr. 2019;13(2):1675-8.

34 Campos JR, Costa FO, Cota LOM. Association between periodontitis and metabolic syndrome: a case-control study. J Periodontol. 2020 Jun;91(6):784-91.

35 Kim JS, Kim SY, Byon MJ, Lee JH, Jeong SH, Kim JB. Association between periodontitis and metabolic syndrome in a Korean nationally representative sample of adults aged 35 79 years. Int J Environ Res Public Health. 2019;16(16):E2930.

36 Sakurai SI, Yamada SI, Karasawa I, Sakurai A, Kurita $\mathrm{H}$. A longitudinal study on the relationship between dental health and metabolic syndrome in Japan. J Periodontol. 2019;90(7): 728-46.

37 Leite FRM, Nascimento GG, Scheutz F, López R. Effect of smoking on periodontitis: a systematic review and meta-regression. Am J Prev Med. 2018;54(6):831-41.
38 Lee EJ, Han KD, Han JH, Lee JH. Smoking and risk of psoriasis: a nationwide cohort study. J Am Acad Dermatol. 2017;77(3):5735.

39 Naldi L. Psoriasis and smoking: links and risks. Psoriasis (Auckl). 2016;6:65-71.

40 Nociti FH Jr, Casati MZ, Duarte PM. Current perspective of the impact of smoking on the progression and treatment of periodontitis. Periodontol 2000. 2015;67(1):187-210.

41 Borojevic T. Smoking and periodontal disease. Mater Sociomed. 2012;24(4):274-6.

42 Johannsen A, Susin C, Gustafsson A. Smoking and inflammation: evidence for a synergistic role in chronic disease. Periodontol 2000. 2014;64(1):111-26.

43 Stolt P, Bengtsson C, Nordmark B, Lindblad S, Lundberg I, Klareskog L, et al. Quantification of the influence of cigarette smoking on rheumatoid arthritis: results from a population based case-control study, using incident cases. Ann Rheum Dis. 2003;62(9):835-41.
44 Ross R. Atherosclerosis - an inflammatory disease. N Engl J Med. 1999;340(2):115-26.

45 van der Heide F, Dijkstra A, Albersnagel FA, Kleibeuker JH, Dijkstra G. Active and passive smoking behaviour and cessation plans of patients with Crohn's disease and ulcerative colitis. J Crohns Colitis. 2010;4(2):125-31.

46 Rawlinson A, Grummitt JM, Walsh TF, Ian Douglas CW. Interleukin 1 and receptor antagonist levels in gingival crevicular fluid in heavy smokers versus non-smokers. J Clin Periodontol. 2003;30(1):42-8.

47 Tymkiw KD, Thunell DH, Johnson GK, Joly S, Burnell KK, Cavanaugh JE, et al. Influence of smoking on gingival crevicular fluid cytokines in severe chronic periodontitis. J Clin Periodontol. 2011;38(3):219-28.

48 Meisel P, Schwahn C, Gesch D, Bernhardt O, John U, Kocher T. Dose-effect relation of smoking and the interleukin-1 gene polymorphism in periodontal disease. J Periodontol. 2004;75(2):236-42. 